\title{
Efeitos adversos da morfina, metadona e tramadol no pós-operatório de cães submetidos à cirurgia da coluna vertebral: 180 casos $(2011-2016)^{1}$
}

\author{
Angel Ripplinger ${ }^{2}$, Graciane Aiello², Rafael O. Chaves², Amanda O. Andrades², \\ Diego V. Beckmann ${ }^{3}$, Dakir Polidoro ${ }^{2}$, André V. Soares ${ }^{4}$ e Alexandre Mazzanti ${ }^{*}$
}

\begin{abstract}
Ripplinger A., Aiello G., Chaves R.O., Andrades A.O., Beckmann D.V., Polidoro D., Soares A.V. \& Mazzanti A. 2018. [Adverse effects of morphine, methadone and tramadol in the postoperative period of dogs undergone vertebral surgery: 180 cases (2011-2016).] Efeitos adversos da morfina, metadona e tramadol no pós-operatório de cães submetidos à cirurgia da coluna vertebral: 180 cães (2011-2016). Pesquisa Veterinária Brasileira 38(7):1431-1437. Departamento de Clínica de Pequenos Animais, Universidade Federal de Santa Maria, Av. Roraima 1000, Camobi, Santa Maria, RS 97105-900, Brazil. E-mail: alexamazza@yahoo.com.br

Postoperative pain in dogs undergone vertebral surgery is classified as severe and it's important an adequate approach to it, because it can influence recovery time, quality of life and surgery outcome. Opioids are indicated for postoperative pain treatment in these surgeries. Opioids may have adverse effects that may require attention. There are few clinical studies that present the adverse effects of these analgesics in canine postoperative period. The aim of this retrospective study was to present the adverse effects of morphine, methadone and tramadol in canine vertebral surgery postoperative period. There were revised the postoperative records of 180 dogs and the changes resulted from the opioids use were noted. The adverse effects observed were anorexia, hyporexia, vomiting, vocalization, bradycardia, hypothermia, panting, sedation. Pain was also observed in some dogs. A significant difference was found in anorexia between dogs treated with morphine and tramadol and methadone and tramadol. Significant difference was also found in pain between dogs treated with morphine and tramadol. The association of metamizole and morphine or metamizole and methadone was not different in relation to the adverse effects. There was also no difference with the dosage variation and the adverse effects. In conclusion, morphine, methadone and tramadol have adverse effects when used for pain control in the postoperative period of dogs submitted to vertebral surgery. Anorexia, hypophagia and emesis were frequent the adverse effects observed with morphine and methadone and, despite tramadol presented less adverse effects, its use may be not beneficial in the studied doses when we consider the degree of pain, however more controlled studies with clinical situation are needed to confirm this.
\end{abstract}

INDEX TERMS: Morphine, methadone, tramadol, postoperative period, dogs, vertebral surgery, opioids, adverse effects, surgery.

RESUMO.- A dor pós-operatória em cães que são submetidos a cirurgias da coluna vertebral é considerada severa e seu manejo inadequado pode influenciar no tempo de recuperação

\footnotetext{
${ }^{1}$ Recebido em 7 de junho de 2017.

Aceito para publicação em 21 de junho de 2017.

${ }^{2}$ Programa de Pós-Graduação em Medicina Veterinária, Universidade Federal de Santa Maria (UFSM), Av. Roraima 1000, Santa Maria, RS 97105-900, Brasil.

${ }^{3}$ Docente da Faculdade de Veterinária, Universidade Federal do Pampa, Rodovia BR-472, Uruguaiana, RS 97508-000, Brasil.

${ }^{4}$ Departamento de Clínica de Pequenos Animais, Centro de Ciências Rurais, Universidade Federal de Santa Maria (UFSM), Av. Roraima 1000, Camobi, Santa Maria, RS 97105-900, Brasil. *Autor para correspondência: alexamazza@yahoo.com.br
}

do paciente, na qualidade de vida e no resultado cirúrgico. Dentre os analgésicos indicados para uso no pós-operatório dessas cirurgias tem-se os opioides, que podem apresentar inúmeros efeitos adversos que requerem atenção. Devido à escassez de estudos clínicos acerca desse assunto em se tratando do pós-operatório de cães, objetivou-se com o presente estudo retrospectivo apresentar os efeitos adversos da morfina, metadona e tramadol utilizados no pós-operatório de cirurgias da coluna vertebral. Foram revisadas e avaliadas as fichas de 180 cães e anotadas as alterações observadas no pós-operatório e decorrentes do uso de opioides. Os principais efeitos adversos observados foram anorexia, hiporexia, vômito, salivação, vocalização, bradicardia, hipotermia, 
ofegação e sedação. Também foi observada persistência da dor em alguns cães mesmo com o uso de analgésicos. Houve diferença na ocorrência de anorexia nos cães tratados com morfina e nos tratados com metadona em relação aos tratados com tramadol. Ocorreu diferença também entre a observação de dor dos grupos morfina e tramadol. A associação de dipirona com morfina e com metadona não revelou diferença com relação à ocorrência de efeitos adversos, bem como a variação de doses. Conclui-se que a morfina, a metadona e o tramadol apresentam efeitos adversos quando empregados para tratamento da dor pós-operatória em cães submetidos à cirurgia da coluna vertebral; a anorexia, a hiporexia e o vômito foram os efeitos adversos frequentes com o uso de morfina e de metadona e, mesmo que o tramadol apresente menor ocorrência desses efeitos, seu uso, na dose estudada, pode não ser vantajoso quando se leva em consideração o grau de dor para cirurgias da coluna vertebral.

TERMOS DE INDEXAÇÃO: Morfina, metadona, tramadol, pós-operatório, cães, coluna vertebral, opioides, efeitos adversos, cirurgia.

\section{INTRODUÇÃO}

O controle da dor pós-operatória é uma preocupação constante, pois o manejo inadequado pode influenciar no tempo de recuperação do paciente, na qualidade de vida e no resultado cirúrgico (Ledowski et al. 2012).

Diversas doenças adquiridas podem causar dor espinhal, incluindo afecções neurológicas e não neurológicas, cujo tratamento deve ser direcionado tanto para a causa como para o alívio do desconforto causado por ela (Platt 2004). A dor espinhal pode ser resultado de lesões que afetam meninges; raízes nervosas; anulo fibroso do disco intervertebral; periósteo vertebral; cápsulas articulares (principalmente aquelas da articulação dos processos intervertebrais); musculatura epaxial e estruturas ligamentosas que circundam a vértebra (Platt 2004, Puvanesarajah et al. 2015).

Sabendo que nessas localizações a quantidade de nociceptores é alta (Hall 2011, Lorenz et al. 2011, Gaynor 2014), faz-se necessário o uso de opioides potentes para controle da dor pós-operatória quando essas estruturas são afetadas (Monteiro et al. 2009, Fantoni \& Mastrocinque 2010, Martins et al. 2010), pois geram dor de nível intenso considerada grave/severa (Fantoni \& Mastrocinque 2010, Mathews et al. 2014).

A morfina é o fármaco referência dos analgésicos opioides, produz adequada analgesia pela alta afinidade com o receptor $\mu$ (Epstein 2015) e é considerado o analgésico de eleição para o tratamento da dor grave (Johnson 1991, Hellyer \& Gaynor 1998, Mathews et al. 2014). A metadona é um opioide sintético $\mu$-agonista (Duke-Novakovski 2014) que foi recentemente introduzido na rotina veterinária para tratamento da dor grave (Cardozo et al. 2014). Sua eficácia intrínseca de ligação com receptores opioides $\mu$ é maior do que a da morfina (Selley et al. 2001) e, além da afinidade pelo receptor opioide $\mu$, relata-se atividade da metadona em receptores N-Metil-D-Aspartato (NMDA) e $\alpha-2$ (Codd et al. 1995). Já o tramadol é um analgésico com uso indicado para tratamento da dor leve a moderada por apresentar um décimo da potência da codeína e 6000 vezes menor que a morfina (Mastrocinque \& Fantoni 2003). Seu efeito se dá por meio da ligação com receptores opioides $\mu$, atuando também como um $\alpha-2$ agonista através da inibição da recaptação neuronal da norepinefrina e da serotonina (Gaynor \& Muir 3rd 2015), sendo por isso classificado como um agonista $\mu$ atípico (Duke-Novakovski 2014). A potencialização do seu efeito se dá mediante associação com anti-inflamatórios não esteroidais (Gaynor \& Muir 3rd 2015).

Os efeitos adversos dos opioides variam conforme a espécie animal e são mais comumente verificados quando da utilização de doses excessivas e incluem vômito, disforia, náusea, ofegação, bradicardia e liberação de histamina (que causa vasodilatação e hipotensão), incontinência ou retenção urinária e depressão respiratória (Mathews et al. 2014, Epstein 2015). A incidência desses efeitos pode variar substancialmente de acordo com o agente, momento da aplicação, bem como número de aplicações (Fantoni \& Mastrocinque 2010).

Muitos estudos pré-clínicos sobre opioides são conduzidos em animais saudáveis, sem ocorrência natural de dor, entretanto os efeitos causados pelos opioides em um paciente com ocorrência natural de dor podem ser diferentes (Kukanich \& Wiese 2015). Estudos clínicos em animais naturalmente com dor, com objetivo principal de verificar a ocorrência de efeitos adversos comparando diferentes fármacos opioides em cães são escassos e, as informações que existem na literatura estão em geral ligadas a apenas um fármaco, sem comparação desses efeitos entre fármacos. Assim o objetivo do presente estudo é relatar os principais efeitos adversos relacionados ao uso de opioides na rotina hospitalar em cães submetidos a cirurgias da coluna vertebral.

\section{MATERIAL E MÉTODOS}

Foram revisados os protocolos para analgesia pós-operatória de cães submetidos à cirurgia da coluna vertebral no Hospital Veterinário Universitário da Instituição entre 2011 e 2016. Dos protocolos analgésicos foram compilados os dados referentes ao tipo de opioide utilizado, dose, frequência, via de administração, associação com outros fármacos empregados no controle da dor, bem como os efeitos adversos observados.

Quanto ao tipo de opioide utilizado os cães foram distribuídos em três grupos, denominados morfina (GMo), metadona (GMe)e tramadol (GT).

As técnicas cirúrgicas utilizadas foram hemilaminectomia dorsolateral, laminectomia dorsal modificada, corpectomia parcial, fenda ventral (slot) ou estabilização de fratura/luxação vertebral mediante uso de pinos de Steimann e cimento ósseo (Sharp \& Wheeler 2005) e variaram de acordo com o tipo de afecção do paciente.

Quanto às anotações de pós-operatório foram considerados efeitos adversos do fármaco: anorexia - perda de apetite (Monroe 2004) percebida como a não ingestão de alimento, seja ração seca, pastosa ou comida caseira; hipofagia ou hiporexia - perda parcial do apetite (Monroe 2004) caracterizada pela ingestão de pouca quantidade de alimento seja ração seca, pastosa ou comida caseira, por parte de um paciente que, internado no pré-operatório, ingeria quantidade de alimento adequada ao seu tamanho; vômito secundário à aplicação do fármaco - quando o paciente não apresentasse outra alteração que justificasse tal evento, como uremia, sendo o vômito caracterizado por processo ativo com sinais prodrômicos e fases de náusea, contrações da parede abdominal improdutivas e ejeção forçada de conteúdo gástrico associada a contrações abdominais fortes e repetitivas (Dossin 2008); sialorreia - salivação excessiva (Willard 2010), sendo que a mesma flui para fora da cavidade oral; sedação - estado caracterizado por depressão central, sonolência 
e algum grau de relaxamento (Tranquilli \& Grimm 2015); náusea - associação de sialorreia, agitação ou depressão, bocejo, lambedura dos lábios e/ou repetidas tentativas de deglutição (Dossin 2008); vocalização contínua ou intermitente - quando o paciente não apresentava essa alteração durante a internação de pré-operatório; hipotermia - temperatura corporal abaixo dos valores aceitos para a espécie, $37,5^{\circ} \mathrm{C}$ para cães (Miller 2004) após completa recuperação anestésica; bradicardia - ritmo cardíaco anormalmente lento, com redução de $20 \%$ do valor basal pré-operatório (Haskins 2015); taquipneia - aumento da frequência respiratória (Turnwald 2004) com respiração superficial e rápida (Ware 2010). Também foram consideradas anotações associadas à dor, mesmo não tendo sido utilizadas escalas de avaliação de dor, quando esta anotação estivesse relacionada à manipulação do paciente envolvendo a área cirúrgica e este apresentasse comportamento de proteção como se esquivar, lamber, rosnar ou tentar morder (Fantoni \& Mastrocinque 2010). A dor foi considerada efeito relacionado à ineficiência do fármaco.

Como critério de inclusão, foram considerados somente cães que tivessem fichas de pós-operatório completas, tempo de internação de pelo menos 36 horas de pós-operatório, pacientes que não apresentassem alterações orgânicas que pudessem levar a quadro que mimetizasse os efeitos adversos dos fármacos utilizados. Embora os anti-inflamatórios façam parte de terapias analgésicas multimodais, os pacientes que receberam anti-inflamatórios no pós-operatório não foram incluídos no estudo, pois em alguns cães o uso desses medicamentos pode causar efeitos colaterais que mimetizem os dos opioides.

Os resultados referentes aos efeitos da medicação no pós-operatório foram comparados entre si mediante teste para diferença de proporções devido ao diferente $\mathrm{N}$ entre os grupos, considerando diferença significativa quando $\mathrm{p}<0,05$. Foram também comparadas entre si pelo teste de diferença de proporções as diferentes doses dos opioides utilizados para verificar se a diferença na dose do medicamento influenciou na ocorrência de efeito colateral, sendo considerado significativo quando $\mathrm{p}<0,05$.

Das 213 fichas revisadas que constavam os protocolos para analgesia pós-operatória de cães submetidos à cirurgia da coluna vertebral, foram incluídas 180 que atenderam aos critérios estabelecidos.

\section{RESULTADOS E DISCUSSÃO}

Todos os procedimentos cirúrgicos verificados neste estudo envolveram a manipulação de estruturas ricas em nociceptores como camadas superficiais da pele, periósteo, paredes arteriais, cápsulas articulares, músculos, tendões e meninges (Platt 2004, Hall 2011, Lorenz et al. 2011) causadoras de dor severa (Fantoni \& Mastrocinque 2010, Mathews et al. 2014), a qual necessita de analgésicos potentes para seu controle. Assim sendo, todos os cães receberam opioides, considerado por Monteiro et al. (2009) e Fantoni \& Mastrocinque (2010) como a classe de analgésicos mais indicada para uso no pós-operatório nessas situações.

Dos 180 cães envolvidos, 104 (57,7\%) receberam como tratamento pós-operatório sulfato de morfina em doses que variaram de 0,3 a $0,8 \mathrm{mg} \cdot \mathrm{kg}^{-1}$ por via subcutânea, $46(25,5 \%)$ cloridrato de tramadol em doses que variaram de 3 a $8 \mathrm{mg} . \mathrm{kg}^{-1}$ por via subcutânea e $30(16,6 \%)$ cloridrato de metadona em doses variando entre 0,3 e $0,5 \mathrm{mg} \mathrm{kg}^{-1}$ por via subcutânea (Fig1.). Os intervalos de aplicação dos fármacos foram de 4 a 6 horas nas primeiras 24 horas de pós-operatório. Todos os pacientes medicados com tramadol receberam concomitantemente dipirona sódica como adjuvante na analgesia. Os pacientes do presente estudo receberam ao término do procedimento uma aplicação de dexametasona $\left(0,25 \mathrm{mg}_{\mathrm{kg}} \mathrm{kg}^{-1}\right)$ não receberam mais aplicações anti-inflamatórios no pós-operatório.

Foi verificado também que 75\% (135/180) dos cães receberam dipirona sódica associada ao opioide como forma de potencializar a analgesia, pois estudos de Tempel et al. (1996), Kemal et al. (2007) e Ohnesorge et al. (2009) notaram que o consumo de morfina foi reduzido pela inclusão da dipirona no protocolo analgésico, além deste fármaco bloquear a dor por uma via diferente dos opioides. A dose utilizada da dipirona sódica foi de 25 mg. $\mathrm{kg}^{-1}$, a cada 8 horas, sendo considerada segura para analgesia sem efeitos adversos, conforme salientaram Imagawa et al. (2011). A associação do opioide com a dipirona sódica, não apresentou diferença na ocorrência dos efeitos adversos tanto nos cães do GMo quanto naqueles do GMe ( $\mathrm{p}=0,94$ e $\mathrm{p}=0,40$, respectivamente).

Dos 104 pacientes tratados com sulfato de morfina, 56,7\% apresentaram efeitos adversos os quais foram anorexia $(33,6 \%)$, hiporexia $(5,7 \%)$, vômito $(5,7 \%)$, vocalização $(1,9 \%)$, taquipneia/ofegação (1,9\%) e sedação, bradicardia e hipotermia (0,9\% cada) (Fig.2). Neste grupo verificou-se persistência da dor em 4,8\% dos cães. Quanto aos 30 pacientes tratados com cloridrato de metadona, 66,6\% apresentaram efeitos adversos, sendo estes anorexia (13\%), vômito (10\%), vocalização (6,6\%), sedação (3,3\%) e, a dor persistiu em3,3\% (Fig.2). Já os 46 pacientes que receberam cloridrato de tramadol como analgésico pós-operatório imediato, 32,6\% apresentaram

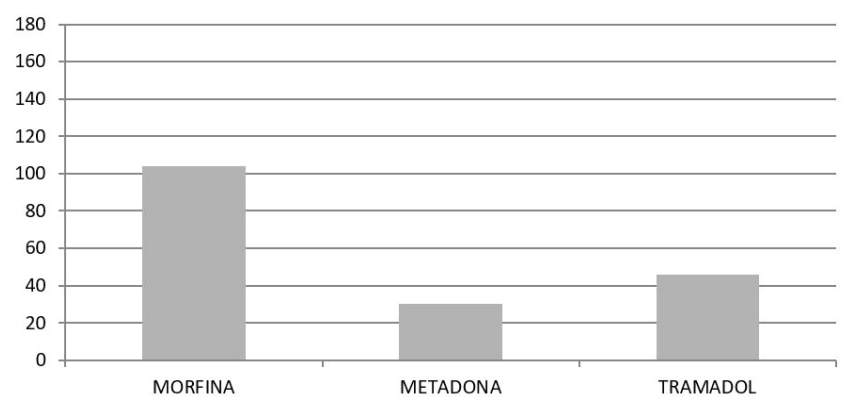

Fig.1. Representação quanto ao tipo de opioide utilizado no pós-operatório de 180 cães submetidos à cirurgia na coluna vertebral.

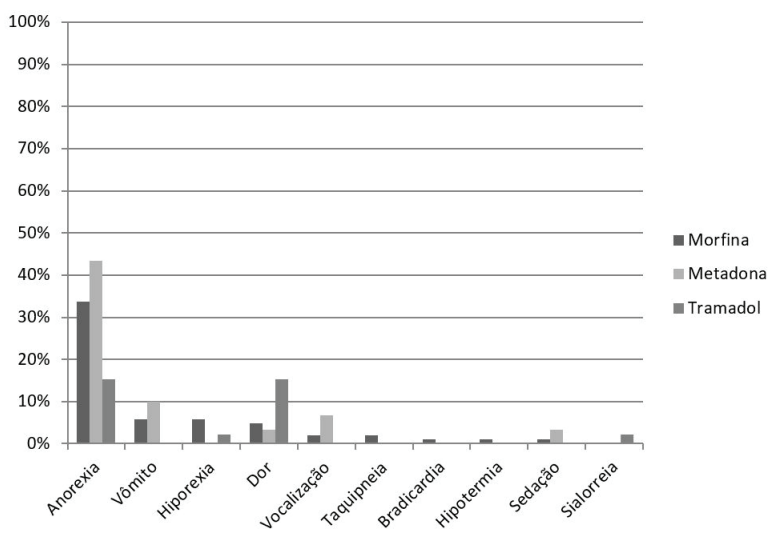

Fig.2. Ocorrência de efeitos adversos dos diferentes tipos de opioides utilizados no pós-operatório de cães submetidos à cirurgia na coluna vertebral. 
efeitos adversos como anorexia $(15,2 \%)$, e sialorreia $(2,1 \%)$, verificando-se dor persistente em 15,2\% dos cães (Fig.2). Embora a literatura cite que a dose dos opioides seja uma das variáveis que interfere na ocorrência ou não de efeitos adversos (Kukanich \& Wiese 2015), no presente estudo a variação nas doses administradas de morfina, metadona e tramadol não influenciaram na ocorrência desses efeitos.

Quando os efeitos adversos apresentados pelos pacientes dos diferentes grupos de tratamento foram comparados entre si, verificou-se que os cães do GMo e GMe apresentaram mais anorexia que o GT $(\mathrm{p}<0,05)$. A metadona é um fármaco que está ganhando cada vez mais adeptos na rotina veterinária pelo fato de apresentar efeito analgésico tão potente quando o da morfina, mas com menor ocorrência de efeitos adversos como náusea, vômito e disforia (Monteiro et al. 2008), entretanto não foi possível comprovar isso no presente estudo retrospectivo, já que não houve diferença entre os efeitos adversos dos grupos tratados com metadona quando comparados ao grupo tratado com morfina.

Os efeitos adversos mais conhecidos da morfina são náusea e vômito, que estão relacionados à estimulação direta dos receptores de dopamina da zona deflagradora dos quimiorreceptores localizados no terceiro ventrículo no sistema nervoso central (Blancquaert et al. 1986). Outro mecanismo que justifica náusea e vômito em cães medicados com opioides é a redução da motilidade propulsiva gastrointestinal (Kukanich \& Wiese 2015). Esses efeitos são geralmente verificados no uso agudo do fármaco, desaparecendo com o uso crônico (Fantoni \& Mastrocinque 2010).

No presente estudo não houve anotações referentes à náusea, mas, pode-se inferir que a este sinal tenha sido a causa da anorexia e mesmo da hipofagia verificadas nos pacientes dos três grupos de tratamento, como também sugeriu Epstein (2015). 0 vômito foi encontrado em 5,7\% dos pacientes tratados com morfina e, acredita-se que doses mais baixas deste fármaco tendem a produzir efeito emético pela estimulação de receptores de dopamina (Blancquaert et al. 1986, Papich 2000, Kukanich \& Wiese 2015). Doses mais altas ou múltiplas de morfina tendem a produzir efeito antiemético (Kukanich \& Wiese 2015).

Discordando da literatura consultada, a qual ressaltou que a morfina causa mais vômito que a metadona e o tramadol (Valverde et al. 2004, Monteiro et al. 2008, Monteiro et al. 2009), verificou-se vômito em $10 \%$ dos cães que receberam a metadona contra 5,7\% dos cães com morfina. Cabe ressaltar aqui que essa diferença não foi estatisticamente significativa. Blancquaert et al. (1986) relataram que a metadona, o fentanil e o butorfanol tendem a produzir efeito antiemético mais proeminente e isso pode estar relacionado a rápida penetração no sistema nervoso central, por serem lipossolúveis, e provocar inibição do centro do vômito, o que é reforçado por Ingvast-Larsson et al. (2010), que, em estudo farmacológico da metadona em cães, não observaram efeitos como vômito ou mesmo agitação. Essa maior ocorrência de vômito no grupo de pacientes estudado quando comparado a outros estudos pode reforçar a hipótese de grande variação individual dos efeitos dos opioides (Kukanich \& Wiese 2015), o que traz um alerta para monitoração mesmo dos pacientes tratados com opioides que tipicamente não trazem esse efeito colateral o qual, precisa ser prevenido ou tratado.
A sialorreia, que sugere presença de náusea (Dossin 2008) foi observada apenas no grupo medicado com tramadol, fato também observado por Cardozo et al. (2014) em um grupo de cães tratado com $4 \mathrm{mg} . \mathrm{kg}^{-1}$ deste fármaco. Essa sialorreia verificada no presente estudo não teve relação com a dose do tramadol. Acredita-se que a via de aplicação do tramadol possa influenciar na ocorrência de efeitos adversos, sendo que salivação e náusea são as mais observadas quando da sua administração pelas vias intramuscular e intravenosa (Kukanich \& Papich 2004, McMillan et al. 2008), reduzindo esses efeitos com a aplicação pela via subcutânea (Buhari et al. 2012).

A vocalização observada nos pacientes que foram tratados com morfina $(1,9 \%)$ e metadona $(6,6 \%)$ pode ser sinal de disforia, a qual, ao contrário da excitação causada pelos opioides pode durar por horas e ser caracterizada por agitação, ataxia, vocalização, desorientação e inquietação (Duke-Novakovski 2014, Kukanich \& Wiese 2015). No caso da metadona, além da vocalização os pacientes podem apresentar choramingos que, para um avaliador descuidado pode ser interpretado como dor, entretanto, esses choramingos são considerados efeitos adversos da própria metadona, principalmente quando administrada por via subcutânea (Ingvast-Larsson et al. 2010).

Sedação é um efeito típico dos opioides em cães livres de dor e, em alguns casos, pode ser considerado útil também no pós-operatório imediato quando o repouso é desejado (Duke-Novakovski 2014, Epstein 2015). Entretanto, a sedação passa a ser prejudicial no pós-operatório quando for acentuada a ponto de interferir com a ingestão de água e alimento (Epstein 2015). Observamos sedação em 0,9\% dos pacientes tratados com morfina e em 3,3\% dos tratados com metadona. Quando se verifica sedação excessiva que possa vir a prejudicar a recuperação do pacientes, indica-se alteração ou diminuição da dose do opioide utilizado a fim de minimizar este efeito já que se considera que o grau de sedação é dose-dependente (Epstein 2015). Não foi verificada sedação nos pacientes que receberam tramadol ou o grau de sedação foi mínimo que não pode ser percebido. Essa diferença quanto à sedação ao comparar tramadol e outros opioides como morfina e metadona já foi identificada e relatada em outros estudos (Cardozo et al. 2014)

Os opioides são geralmente usados em pacientes com instabilidade cardiovascular já que, em doses clínicas, tem mínimos efeitos no rendimento e ritmo cardíaco e pressão arterial (Duke-Novakovski 2014). A bradicardia verificada apenas em 1,9\% dos pacientes em que foi administrada morfina é um efeito que pode ocorrer em cães medicados com opioides como resultado da estimulação de núcleos vagais, entretanto tal efeito não é irreversível (Duke-Novakovski 2014, Kukanich \& Wiese 2015). Reduções na frequência cardíaca foram verificadas também quando do uso da metadona em cães saudáveis e alertas (Maiante et al. 2009, Garofalo et al. 2012). A possibilidade da ocorrência de bradicardia inspira maiores cuidados para com os pacientes sob tratamento com opioides. A hipotensão, outro efeito cardiovascular preocupante nos períodos pós-operatórios especialmente nos pacientes que passam por cirurgia que envolve a coluna vertebral não consta no presente estudo por não ser mensurado rotineiramente e, cita-se como de maior ocorrência quando do uso de altas doses de morfina pela via intravenosa a qual causa liberação de histamina, vasodilatação e consequente hipotensão (Kalthum \& Waterman 1988). 
Embora o presente estudo tenha verificado hipotermia apenas em uma baixa porcentagem $(0,9 \%)$ dos pacientes do grupo morfina, a literatura apresenta o potencial dos opioides interferirem na termorregulação dos cães. Acredita-se que isso ocorra pela interação direta com neurônios no hipotálamo anterior pré-óptico alterando o ponto de ajuste termorregulatório bem como respostas compensatórias (Adler et al. 1988, Pascoe 2000). A ofegação secundária ao uso de opioides, verificada em 1,9\% dos cães tratados com morfina no presente estudo, está relacionada à redefinição da temperatura mínima corporal no centro termorregulatório no tálamo, o que faz com que o cão normotérmico perceba calor e fique ofegante como forma de se refrescar (Epstein 2015).

Não se notou, no presente estudo, efeitos adversos dos opioides no sistema respiratório. 0 efeito mais importante e mais preocupante dos opioides no sistema respiratório é a depressão respiratória. Embora humanos sejam mais susceptíveis à depressão respiratória induzida por opioides (Pattinson 2008), a vigilância deve ser mantida mesmo com o uso em cães, pois pode ocorrer também nesta espécie dependendo da via de administração e da dose utilizada do opioide (Monteiro et al. 2009, Garofalo et al. 2012). Acredita-se que a dor estimule o centro respiratório e por fim compense a depressão respiratória causada por opioides em animais (Pattinson 2008).

A dor pode ser considerada ineficiência do opioide (Epstein 2012). Comportamentos que sugeriam persistência da dor foram verificados com todos os protocolos de tratamentos do presente estudo retrospectivo. Houve diferença estatística quando comparado o GMo e o GT ( $\mathrm{p}<0,05)$, ou seja, o tramadol, mesmo associado à dipirona, foi menos eficiente para o grau de dor enfrentado por pacientes após cirurgia da coluna vertebral na dose estudada. Embora o tramadol seja classificado como um opioide atípico e seja reconhecida sua eficácia analgésica em humanos, ele não é indicado para o controle da dor severa em animais, sendo mais indicado como parte de um protocolo de analgesia multimodal (Gaynor \& Muir 3rd 2015).

0 principal metabólito do tramadol reconhecidamente responsável pela analgesia em nível de receptores opioides é o 0-desmetiltramadol (M1) (Kukanich \& Papich 2004, 2011). A produção deste metabólito em cães é realizada pelo grupo de enzimas hepáticas citocromo P450, subtipo 2D15 a qual sofre polimorfismo individual em cães, fazendo com que a produção deste metabólito apresente uma grande variação individual nessa espécie (Giorgi et al. 2010, Perez et al. 2016), o que faz com que a concentração circulante do M1 em cães seja menor do que em humanos e gatos o que justifica menor eficiência analgésica (Perez et al. 2016), além de explicar também a diferença individual quanto aos efeitos do tramadol em diferentes cães (McMillan et al. 2008, Kogel et al. 2014, Goudie-Deangelis \& Woodhouse 2015), o que provavelmente acontece na prática clínica e refletiu no presente estudo. Embora alguns estudos comprovaram a eficácia analgésica do tramadol no pós-operatório de diferentes tipos de cirurgia (Yazbek \& Fantoni 2005, Buhari et al. 2012), a sua real aplicação, nas doses aqui estudadas, no pós-operatório de cirurgias que envolvem a coluna vertebral ainda é questionável, necessitando de estudos controlados direcionados à avaliação de dor após esse tipo de procedimento em cães.

A ocorrência natural de dor pode reduzir alguns dos efeitos indesejados dos opioides em cães, mas é preciso lembrar que não é apenas a presença ou não de dor que tem influência sobre isso (Epstein 2015, Kukanich \& Wiese 2015). Embora os efeitos e doses comuns dos opioides sejam conhecidos é preciso observar a variabilidade dos seus efeitos numa população de cães. Esta variabilidade pode estar relacionada às diferenças farmacodinâmicas e farmacocinéticas individuais, ao comportamento do paciente, genética, sexo além de diferenças raciais. A variabilidade é esperada também em pacientes com diferentes causas de dor (Kukanich \& Wiese 2015).

Em humanos, os sete principais efeitos adversos relatados dos opioides são constipação, náusea persistente, tontura e vertigem, sonolência, vômito, pele seca e prurido, mioclonia e retenção urinária (Epstein 2012). Já em animais, informações precisas não estão disponíveis, entretanto, Epstein (2012) relatou que, de um grupo de veterinários consultados, $20 \%$ relataram observar efeitos adversos dos opioides em seus pacientes e, informalmente, os efeitos reportados foram disforia (41\%), inapetência persistente com ou sem náusea (15\%), íleo e constipação (13\%); ineficácia da medicação (7\%) e depressão respiratória (3\%). Esses dados nos levam a reconhecer a necessidade de mais estudos sobre o assunto, de forma que as limitações encontradas no presente estudo retrospectivo possam ser superadas.

A relevância deste estudo foi reconhecer que os efeitos adversos dos opioides podem ocorrer também em pacientes com dor e que tais efeitos precisam ser tratados, ou pelo menos amenizados para que não causem prejuízo aos pacientes, como a baixa ingestão alimentar e hídrica, secundária à náusea, que, pode prolongar o período de internação e de recuperação. Levando em conta as diferenças individuais de cada paciente, o médico veterinário precisa estar atento à ocorrência de efeitos indesejados mesmo quando utilizados fármacos em que se esperem menos efeitos. Por ser um estudo retrospectivo é necessário reconhecer que apresenta limitações de resultados devido a fatores não controlados.

\section{CONCLUSÕES}

De acordo com os resultados encontrados pode-se concluir que a morfina, a metadona e o tramadol apresentam efeitos adversos quando utilizados no tratamento da dor pós-operatória em cães submetidos à cirurgia da coluna vertebral; a anorexia, a hipofagia e o vômito foram os efeitos adversos frequentes com o uso de morfina e de metadona.

Mesmo que o tramadol apresente menor ocorrência de efeitos adversos, seu uso, no intervalo de doses estudado, pode não ser vantajoso quando se leva em consideração o grau de dor para cirurgias da coluna vertebral.

\section{REFERÊNCIAS}

Adler M.W., Geller E.B., Rosow C.E. \& Cochin J. 1988. The opioid system and temperature regulation. Ann. Rev. Pharmacol. Toxicol. 28(1):429-449.<http:// dx.doi.org/10.1146/annurev.pa.28.040188.002241> <PMid:2837979>

Blancquaert J.P., Lefebvre R.A. \& Willems J.L. 1986. Emetic and antiemetic effects of opioids in the dog. European J. Pharmacol. 128(3):143-150. <http://dx.doi.org/10.1016/0014-2999(86)90760-0><PMid:3024991>

Buhari S., Hashim K., Yong Meng G., Mustapha N.M. \& Gan S.H. 2012. Subcutaneous administration of tramadol after elective surgery is as effective as intravenous administration in relieving acute pain and inflamation in dogs. Scient. World J. 2012:1-7.<http://dx.doi.org/10.1100/2012/564939> 
Cardozo L.B., Cotes L.C., Kahvegian M.A., Rizzo M.F., Otsuki D.A., Ferrigno C.R. \& Fantoni D.T. 2014. Evaluation of the effects of methadone and tramadol on postoperative analgesia and sérum interleukin- 6 in dogs undergoing orthopaedic surgery. BMC Vet. Res. 10(1):1-7.<http://dx.doi.org/10.1186/ s12917-014-0194-7><PMid:25193623>

Codd E.E., Shank R., Schupsky J.J. \& Raffa R.B. 1995. Serotonin and norepinephrine uptake inhibiting activity of centrally acting analgesics: structural determinants and role in antinociception. J. Pharmacol. Exptl Therapeutics 274(3):1263-1270. <PMid:7562497>

Dossin 0. 2008. Diagnostic tools: clinical history, p.3-9. In: Steiner J.M. (Ed), Small animal gastroenterology. Schlütersche Verlag, Hasnnover.

Duke-Novakovski T. 2014. Opioids, p.41-67. In: Egger C.M., Love L. \& Doherty T. (Eds), Pain management in veterinary practice. Wiley Blackwell, Ames.

Epstein M.E. 2012. Opioids: a practical guide and new developments. North American Veterinary Conference, Orlando.

Epstein M.E. 2015. Opioids, p.161-195. In: Gaynor J.S. \& Muir III W.W. (Eds), Handbook of Veterinary Pain Management. Elsevier, St Louis. <http:// dx.doi.org/10.1016/B978-0-323-08935-7.00009-0>.

Fantoni D.T. \& Mastrocinque S. 2010. Fisiopatologia e controle da dor aguda, p.522-544. In: Fantoni D.T. \& Cortopassi S.R.G. (Eds), Anestesia em Cães e Gatos. Roca, São Paulo.

Garofalo N.A., Teixeira Neto F.J., Pereira C.D., Pignaton W., Vicente F. \& Alvaides R.K. 2012. Cardiorespiratory and neuroendocrine changes induced by methadone in conscious and in isuflurane anaesthetised dogs. Vet. J. 194(3):398-404. <http://dx.doi.org/10.1016/j.tvjl.2012.03.019> $<$ PMid:22750283>

Gaynor J.S. 2014. Fisiologia da dor e princípios para seu tratamento, p.52-63. In: Bojrab M.J. (Ed.), Mecanismos das Doenças em Cirurgia de Pequenos Animais. Roca, São Paulo.

Gaynor J.S. \& Muir 3rd W.W. 2015. Alternative drugs and novel therapies used to treat pain, p.280-301. In: Gaynor J.S. \& Muir III W.W. (Eds), Handbook of Veterinary Pain Management. Elsevier, St Louis. <http://dx.doi.org/10.1016/ B978-0-323-08935-7.00014-4>.

Giorgi M., Del Carlo S., Lebkowska-Wieruszewska B., Kowalski C.J. \& Saccomanni G. 2010. Pharmacokinetics of tramadol and metabolites after injective administrations in dogs. Polish J. Vet. Sci. 13(4):639-644. <http://dx.doi. org/10.2478/v10181-010-0027-y><PMid:21370741>

Goudie-Deangelis E.M. \& Woodhouse K.J. 2015. Analgesic efficacy and associated plasma concentration of tramadol and 0-desmethyltramadol following oral administration post ovariohysterectomy in dogs. Peerj PrePrints 3(1)1-25.

Hall J.E. 2011. Sensações somáticas II: dor, cefaleia e sensações térmicas, p.617-628. In: Ibid. (Ed.), Guyton e Hall: Tratado de Fisiologia Médica. Elsevier, Rio de Janeiro.

Haskins S.C. 2015. Monitoring anesthetized patients, p.86-113. In: Grimm K.A., Lamont L.A., Tranquili W.J., Greene S.A. \& Robertson S.A. (Eds), Lumb and Jones Veterinary Anesthesia and Analgesia. 5th ed. Wiley, Blackwell.

Hellyer P.W. \& Gaynor J.S. 1998. Acute postsurgical pain in dogs and cats. Comp. Cont. Educ. Small Anim. Pract. 20(2):140-154.

Imagawa V.H., Fantoni D.T., Tatarunas A.C., Mastrocinque S., Almeida T.F., Ferreira F. \& Posso I.P. 2011. The use of diferente doses of metamizol for post-operative analgesia in dogs. Vet. Anaesth. Analg. 38(4):385-393. <http://dx.doi.org/10.1111/j.1467-2995.2011.00617.x><PMid:21627755>

Ingvast-Larsson C., Holgersson A., Bondesson U., Lagerstedt A.S. \& Olsson K. 2010. Clinical pharmacology of methadone in dogs. Vet. Anaesth. Analg. 37(1):48-56. <http://dx.doi.org/10.1111/j.1467-2995.2009.00476.x> $<$ PMid:20017819>

Johnson J. 1991. The veterinarian's responsability: assessing and managing acute pain in dogs and cats. Comp. Cont. Educ. Small Anim. Pract. 13:911-921.
Kalthum W. \& Waterman A.E. 1988. The pharmacokinetics of intravenous pethidine $\mathrm{HCl}$ in dogs: normal and surgical cases. J. Assoc. Vet. Anaesthetists 15:39-54.

Kemal S.O., Sahin S. \& Apan A. 2007. Comparison of tramadol, tramadolmetamizol and tramadol-lornoxicam administered by intravenous PCA in management of postoperative pain. Agriculture 19(4):24-31. <PMid:18159576>

Kögel B., Terlinden R. \& Schneider J. 2014. Characterisation of tramadol, morphine and tapentadol in an acute pain model in Beagle dogs. Vet. Anaesth. Analg. 41(3):297-304.<http://dx.doi.org/10.1111/vaa.12140> <PMid:24576316>

Kukanich B. \& Papich M.G. 2004. Pharmacokinetics of tramadol and the metabolite 0-desmethyltramadol in dogs. J. Vet. Pharmacol. Therapeutics 27(4):239-246. <http://dx.doi.org/10.1111/j.1365-2885.2004.00578.x> $<$ PMid:15305853>

Kukanich B. \& Papich M.G. 2011. Pharmacokinetics and antinociceptive effects of oral tramadol hydrochloride administration in Greyhounds. Am. J. Vet. Res. 72(2):256-262. <http://dx.doi.org/10.2460/ajvr.72.2.256> <PMid:21281202>

Kukanich B. \& Wiese A.J. 2015. Opioids, p.207-226. In: Grimm K.A., Lamont L.A., Tranquili W.J., Greene S.A. \& Robertson S.A. (Eds), Lumb and Jones Veterinary Anesthesia and Analgesia. 5th ed. Wiley, Blackwell.

Ledowski T., Reimer M., Chavez V., Kapoor V. \& Wenk M. 2012. Effects of acute postoperative pain on catecholamine plasma levels, hemodynamic parameters, and cardiac autonomic control. Pain 153(4):759-764. <http:// dx.doi.org/10.1016/j.pain.2011.11.002> <PMid:22305628>

Lorenz M.D., Coates J.R. \& Kent M. 2011. Pain, p.413-431. In: Ibid. (Eds), Handbook of veterinary neurology. Elsevier, St Louis. <http://dx.doi. org/10.1016/B978-1-4377-0651-2.10014-1>.

Maiante A.A., Teixeira Neto F.J., Beier S.L., Corrente J.E. \& Pedroso C.E. 2009. Comparison of the cardio-respiratory effects of methadone and morphine in conscious dogs. J. Vet. Pharmacol. Therapeutics 32(4):317-328. <http:// dx.doi.org/10.1111/j.1365-2885.2008.01042.x><PMid:19614836>

Martins T.l., Kahvegian M.A., Noel-Morgan J., Leon-Román M.A., Otsuki D.A. \& Fantoni D.T. 2010. Comparison of the effects of tramadol, codein, and ketoprofen alone or in combination on postoperative pain and on concentrations of blood glucose, serum cortisol, and serum interleukin-6 in dogs undergoing maxillectomy or mandibulectomy. Am. J. Vet. Res. 71(9):1019-1026. <http:// dx.doi.org/10.2460/ajvr.71.9.1019><PMid:20807140>

Mastrocinque S. \& Fantoni D.T. 2003. A comparison of preoperative tramadol and morphine for control of early postoperative pain in canine ovariohysterectomy. Vet. Anaesth. Analg. 30(4):220-228. <http://dx.doi. org/10.1046/j.1467-2995.2003.00090.x><PMid:12925179>

Mathews K., Kronen P.W., Lascelles D., Nolan A., Robertson S., Steagall P.V., Wright B. \& Yamashita K. 2014. Guidelines for recognition, assessment and treatment of pain. J. Small Anim. Pract. 55(6):E10-E68. <http://dx.doi. org/10.1111/jsap.12200> <PMid:24841489>

McMillan C.J., Livingston A., Clark C.R., Dowling P.M., Taylor S.M., Duke T. \& Terlinden R. 2008. Pharmacokinetics of intravenous tramadol in dogs. Can. J. Vet. Res. 72(4):325-331. <PMid:18783021>

Miller J.B. 2004. Hipertermia e hipotermia, p.6-10. In: Ettinger S.J. \& Feldman E.C. (Eds), Tratado de Medicina Interna Veterinária: doenças do cão e do gato. $5^{\mathrm{a}}$ ed. Guanabara Koogan, Rio de Janeiro.

Monroe W.E. 2004. Anorexia, p.106-108. In: Ettinger S.J. \& Feldman E.C. (Eds). Tratado de Medicina Interna Veterinária: doenças do cão e do gato. $5^{\underline{a}}$ ed. Guanabara Koogan, Rio de Janeiro.

Monteiro E.R., Figueroa C.D.N., Choma J.C., Campagnol D. \& Bettini C.M. 2008. Effects of methadone, alone or in combination with acepromazine or xylazine, on sedation and physiologic values in dogs. Vet. Anaesth. Analg. 35(6):519-527. <http://dx.doi.org/10.1111/j.1467-2995.2008.00412. $\mathrm{x}><$ PMid:18565203> 
Monteiro E.R., Junior A.R., Assis H.M., Campagnol D. \& Quitzan J.G. 2009. Comparative study on the sedative effects of morphine, methadone, butorphanol or tramadol, in combination with acepromazine, in dogs. Vet. Anaesth. Analg. 36(1):25-33.<http://dx.doi.org/10.1111/j.1467-2995.2008.00424. x><PMid:19121156>

Ohnesorge H., Bein B., Hanss R., Francksen H., Mayer L., Scholz J. \& Tonner P.H. 2009. Paracetamol versus metamizol in the treatment of postoperative pain after breast surgery: a randomized, controlled trial. Eur. J. Anaesthesiol. 26(8):648-653. <http://dx.doi.org/10.1097/EJA.0b013e328329b0fd> $<$ PMid:19487950>

Papich M.G. 2000. Pharmacologic considerations for opiate analgesic and nonsteroidal anti-inflammatory drugs. Vet. Clin. N. Am., Small Anim. Pract. 30(4):815-837. <http://dx.doi.org/10.1016/S0195-5616(08)70009-3> $<$ PMid:10932827>

Pascoe P.J. 2000. Opioid analgesics. Vet. Clin. N. Am., Small Anim. Pract. 30(4):757-772. <http://dx.doi.org/10.1016/S0195-5616(08)70005-6>

Pattinson K.T.S. 2008. Opioids and the control of respiration. Brit. J. Anaesthesia 100(6):747-758. <http://dx.doi.org/10.1093/bja/aen094> $<$ PMid:18456641>

Perez T.E., Mealey K.L., Grubb T.L., Greene S.A. \& Court M.H. 2016. Tramadol metabolismo to O-desmethy tramadol (M1) and N-desmethyl tramadol (M2) by dog liver microssomes: species comparison and identification of responsible canine cytochrome P450s (CYPs). Drug Metabolism and Disposition 44(12):1963-1972.<http://dx.doi.org/10.1124/dmd.116.071902>

Platt S.R. 2004. Neck and back pain, p.202-213. In: Platt S.R. \& Olby N.J. (Eds), BSAVA manual of canine and feline neurology. BSAVA, Waterwells.

Puvanesarajah V., Liauw J.A., Lo S., Lina I.A., Witham T.F. \& Gottschalk A. 2015. Analgesic therapy for major spine surgery. Neurosurg. Rev. 38(3):407418, discussion 419. <http://dx.doi.org/10.1007/s10143-015-0605-7> $<$ PMid:25680636>

Selley D.E., Cao C.C., Sexton T., Schwegel J.A., Martin T.J. \& Childers S.R. 2001. $\mathrm{Mu}$-Opioid receptor-mediated G-protein activation by heroin metabolites: evidence for greater efficacy of 6 monoacetylmorphine compared with morphine. Biochem. Pharmacol. 62(4):447-455. <http://dx.doi.org/10.1016/ S0006-2952(01)00689-X><PMid:11448454>

Sharp N.J.H. \& Wheeler S. 2005. Small Animal Spinal Disorders: diagnosis and surgery. Elsevier Mosby, Toronto. 375p.

Tempel G., Von Hundelshausen B. \& Reeker W. 1996. The opiate-sparing effect of dipyrone in post-operative pain therapy with morphine using a patient-controlled analgesic system. Intensive Care Med. 22(10):1043-1047. <http://dx.doi.org/10.1007/BF01699225><PMid:8923067>

Tranquilli W.J. \& Grimm K.A. 2015. Introduction: use, definitions, history, concepts, classification, and considerations for anesthesia and analgesia, p.3-10. In: Grimm K.A., Lamont L.A., Tranquili W.J., Greene S.A. \& Robertson S.A. (Eds), Lumb and Jones Veterinary Anesthesia and Analgesia. 5th ed. Wiley, Blackwell. <http://dx.doi.org/10.1002/9781119421375.ch1>.

Turnwald G.H. 2004. Dispneia e taquipneia, p.172-176. In: Ettinger S.J. \& Feldman E.C. (Eds), Tratado de Medicina Interna Veterinária: doenças do cão e do gato. 5th ed. Guanabara Koogan, Rio de Janeiro.

Valverde A., Cantwell S., Hernández J. \& Brotherson C. 2004. Effects of acepromazine on the incidence of vomiting associated with opioid administration in dogs. Vet. Anaesth. Analg. 31(1):40-45. <http://dx.doi. $\operatorname{org} / 10.1111 / \mathrm{j} .1467-2995.2004 .00128 . \mathrm{x}><$ PMid:14756752>

Ware W.A. 2010. Manifestações clínicas das cardiopatias: o exame do sistema cardiovascular, p.3-11. In: Nelson R.W. \& Couto C.G. (Eds), Medicina Interna de Pequenos Animais. 4th ed. Elsevier, Rio de Janeiro.

Willard M.D. 2010. Manifestações clínicas dos distúrbios gastrointestinais: disfagia, halitose, sialorreia, p.351-353. In: Nelson R.W. \& Couto C.G. (Eds), Medicina Interna de Pequenos Animais. 4th ed. Elsevier, Rio de Janeiro.

Yazbek K.V.B. \& Fantoni D.T. 2005. Evaluation of tramadol, an "atypical" opioid analgesic in the control of immediate postoperative pain in dogs submitted to orthopedic surgical procedures. Braz. J. Vet. Res. Anim. Sci. $42(4): 250-258$ 